\title{
A novel chiral nano structure for optical activities and negative refractive index ${ }^{\text {th }}$
}

\author{
Xiuli Jia ${ }^{\mathrm{a}}$, Qingxin Meng ${ }^{\mathrm{a}}$, Chengxun Yuan ${ }^{\mathrm{a}}$, Zhongxiang Zhou ${ }^{\mathrm{a}}$, Xiaoou \\ Wang ${ }^{\mathrm{a}, *}$ \\ ${ }^{a}$ Harbin institute of technology, Harbin 150001, China
}

\begin{abstract}
We have numerically demonstrated a chiral nano structure based on double layered asymmetric $\mathrm{Au}$ film with hollowed-out split ring resonators on either side of the polyimide. This novel chiral nano structure can form multiple electric dipoles and magnetic dipoles in infrared and visible regions. And the interaction of these dipoles caused optical activities and multi-band negative refractive index. The appropriate geometric parameters are obtained through optimization and analysis of this structure. Circular dichroism, polarization azimuthal rotation angle, ellipticity, refractive index and chiral parameter are calculated using S-parameter retrieval method, and these optical properties can be tunable by the geometric parameters of the chiral structure. This chiral structure metamaterial would offer electromagnetic applications in the infrared and visible regions, for fields like imaging, invisibility cloak and perfect lensing.
\end{abstract}

Keywords: Metamaterial, Nanomaterials, Chiral, Optical activities, Negative refractive index, S-parameter retrieval method.

2015 MSC: 00-01, 99-00

\footnotetext{
National Natural Science Foundation of China (No 61205011), National Science Foundation of China (NSFC) (No. 61205093).

${ }^{*}$ Corresponding author

Email address: wxo@hit.edu.cn (Xiaoou Wang)
} 


\section{Introduction}

Metamaterials with negative refraction have drawn much attention since Smith et al. successfully prepared such metamaterials in the laboratory in 2000. Ref. 1] Recently, increasing attention has been focused on chiral metamateri5 als (CMMs) owing to their attractive electromagnetic (EM) properties, such as their large optical activity, strong circular dichroism (CD) effect, negative refractive index (NRI), and asymmetric transmission effect of linearly polarized waves. Ref. [2, 3, 4, 5, 6, 7, 8, 9, 10, 11] CMMs with self-polarization and cross-polarization between electromagnetic fields can achieve a negative index of refraction depending on their chirality, whether or not the permittivity and permeability are negative. Furthermore, they can exhibit strong optical activity and lower loss of the electromagnetic wave (EMW) transmission. Ref. [3]

Recently, negative refraction CMMs have shown large optical activities (e.g., $\left.\theta=130^{\circ}\right)$, Ref. [12] high frequency regions $\left(3 \times 10^{14} \mathrm{~Hz}\right)$, Ref. [13] lower loss

$15 \quad(\mathrm{FOM}=4.2)$, Ref. [14] and high negative refraction value $(\mathrm{n}=-180)$. Ref. [15] Chirality is the lack of the internal mirror symmetry of a molecule or artificial structure. Optical activity is a major characteristic of CMMs. In nature, optical activity is caused by the intrinsic spiral feature of chiral molecules or the spiral arrangement of its atomic molecules. The optical activity of a natural chiral medium is caused by the intrinsic helical characteristics of molecules or the spiral alignment of atoms, and the root of the rotation is circular birefringence, and strength is very weak. For artificial CMMs, optical activity is caused by the optical spatial dispersion of structural chirality. Compared with nature, CMMs have higher optical activities and chiralities. Ref. [3, 12, 16, 17]

$\mathrm{CD}(\Delta)$ refers to one of the main physical parameters for characterizing the absorption of the RCP wave (+, right circular polarization wave) and the LCP wave (-, left circular polarization wave):

$$
\Delta=\left|T_{++}\right|-\left|T_{--}\right| .
$$


The polarization azimuthal rotation angle $(\eta)$ refers to the angle of rotation (with respect to the plane of polarization) of incident light per unit length:

$$
\theta=\frac{\arg \left(T_{--}\right)-\arg \left(T_{++}\right)}{2}
$$

30 and incident waves, and also measures the CD effect. It is caused by electromagnetic coupling between two layers of the CMM:

$$
\eta=\frac{1}{2} \arctan \left(\frac{\left|T_{++}\right|^{2}-\left|T_{--}\right|^{2}}{\left|T_{++}\right|^{2}+\left|T_{--}\right|^{2}}\right)
$$

Here, $T_{++}$refers to the transmission coefficient of the RCP wave and $T_{--}$ refers to the transmission coefficient of the LCP wave. Using the retrieval meth35 ods for CMMs, Ref. [18] we can obtain the effective impedance $\left(Z_{\text {eff }}\right)$, the refractive index of the RCP and LCP waves $\left(n_{ \pm}\right)$, and the effective refractive index $\left(n_{e f f}\right)$ of the chiral medium:

$$
\begin{gathered}
Z_{e f f}=\sqrt{\frac{(1+R)^{2}-T_{++} T_{--}}{(1-R)^{2}-T_{++} T_{--}}} \\
n_{ \pm}=\frac{-j}{k_{0} d} \ln \left[\frac{1}{T \pm \pm}\left(1-\frac{Z_{e f f}-1}{Z_{e f f}+1} R\right)\right] \\
n_{e f f}=\left(n_{+}+n_{-}\right) / 2
\end{gathered}
$$

Here, $\kappa_{0}$ refers to the wave number in free space, and $d$ refers to the equivalent thickness of the chiral structure. The effective impedance and equivalent refractive index are the basis for obtaining the chiral parameter $\left(\kappa_{e f f}\right)$ : Ref.
18

$$
\kappa_{e f f}=\left(n_{+}-n_{-}\right) / 2
$$

In this work, we used the finite difference time domain (FDTD) method to systematically study the complementary split-ring-shaped CMM. In this context, it has been shown that an NRI and a large optical activity occur at in- 
45 frared and visible light frequencies. The geometrical parameters are examined to explore the strong optical activity, CD effect, and NRI at these frequencies. Endowed with these rich EM (optical) properties, CMMs may lead to many applications in photonic devices on account of their strong optical activity (polarization rotation effect) and CD effect.

\section{PHYSICAL MODEL AND MECHANISM ANALYSIS}

Babinets principle was applied to the design of the metamaterial, resulting in both a complementary spectral response and fields. Ref. [19, 20, 21, 22] We created a chiral structure in an array of conjugated bilayer metal split-ring resonators, as shown in Fig. 1, where the conjugated bilayer metal split-ring resonator is depicted as yellow, and the dielectric layer is depicted as blue. The unit size of the chiral structure is $\mathrm{x}=\mathrm{y}=500 \mathrm{~nm}$. The CMM consists of three layers (i.e., Au/polyimide/Au). The metal layer thickness is represented by $t$, the dielectric layer thickness by $d$, the width of the ring by $g$ (outer radius minus inner radius), and the angle of cut by $\alpha$. The location of the cut rotates by $90^{\circ}$ from ring to ring. The dielectric constant of the polyimide is 3.5 , and the loss tangent is $\tan \delta=0.003$. Gold can be described by Drude dispersion model as follows: $\varepsilon=1-\omega_{p} / \omega\left(\omega+i \omega_{\gamma}\right)$. The plasma frequency is $\omega_{p}=1.37 \times 10^{16} \mathrm{~s}^{-1}$, and the collision frequency is $\omega_{\gamma}=2.04 \times 10^{14} \mathrm{~s}^{-1}$. Ref. 23] The front and back metal layers are shown in left portion of Fig. 1. The back metal layer is obtained by rotating the front layer by $180^{\circ}$ around the Y-axis.

The current density distributions and electric field distributions were obtained from numerical simulations at nine resonance frequencies where strong optical activity occurs, as shown by Fig. 2 . 


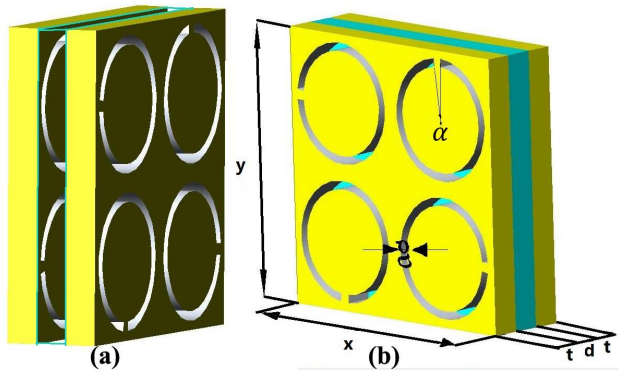

Fig. 1. The unit cell of the chiral metamaterial (CMM). (a) Perspective view. (b) Full dimensions.

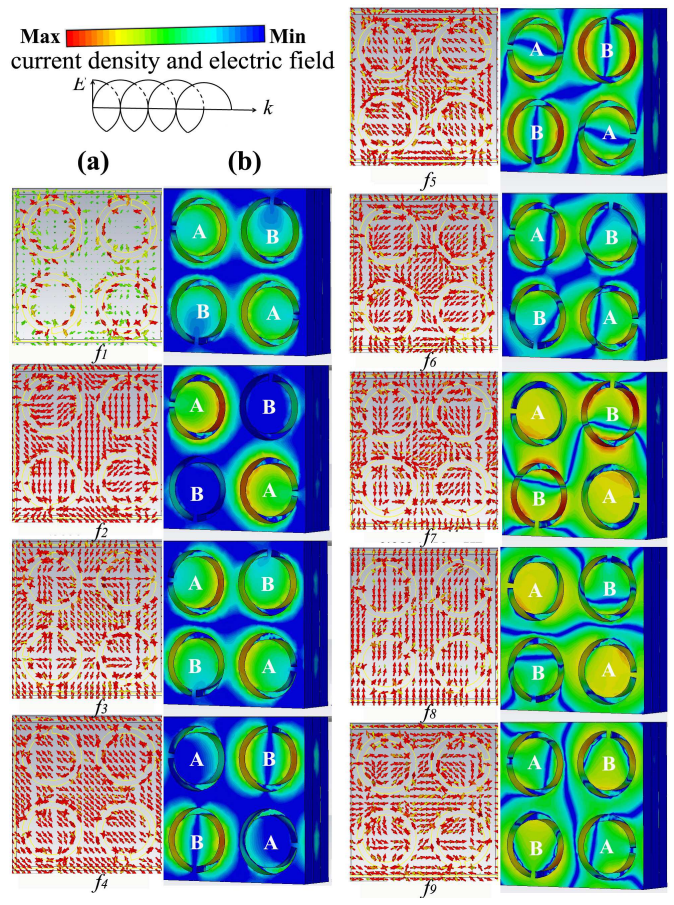

Fig. 2. (a) Distributions of the surface current density. (b) Electric field at nine resonance frequencies.

For the multi-layer chiral structure, strong coupling was found between each 70 layer of metal, which formed symmetric and asymmetric current distributions 
on the surface of the metals. This also generated a strong coupling between the electric and magnetic field, as well as a large chiral parameter, thereby achieving negative refraction. The metal surfaces form multiple current density distribution areas are located inside and outside of the split rings, which can

75 be divided into two regions: the parallel direction region, forming an electric dipole, and the anti-parallel direction region, forming a magnetic dipole, as shown in Fig. 2(a). This mechanism of resonance is different from that of the single electric or magnetic dipole of the cross chiral structure. Ref. 24] Thus, the multiple resonance frequencies are result from the interaction of multiple electric and magnetic dipoles. Interplay between the adjacent electromagnetic dipoles is an experimental priority in metamaterial design. It is an important factor determining the chirality of the structure, optical activities and negative refractive index.

To gain a better understanding of the multiple resonance frequency mecha85 nism, we also calculated the electric field distributions in Fig. 2(b). The electric field strength increases with the frequency. Through an analysis of the electric field under multiple resonance frequencies, we believe that there are two main reasons for this: 1) the CMM structure unit itself produces different resonance frequencies; 2) with the increase of the resonance frequency, the interaction 90 increases between the adjacent units also produces some resonance frequencies.

The electric field strength distribution is calculated for well understanding the coupling enhancement mechanism. Fig. 2(b) depicts the electric field distribution with SRRs structures at $\left(f_{1}\right.$ to $\left.f_{9}\right) 1.7378 \times 10^{14} \mathrm{~Hz}, 2.3599 \times 10^{14} \mathrm{~Hz}$, $3.8499 \times 10^{14} \mathrm{~Hz}, 4.3562 \times 10^{14} \mathrm{~Hz}, 4.9638 \times 10^{14} \mathrm{~Hz}, 6.2224 \times 10^{14} \mathrm{~Hz}, 6.6854 \times 10^{14}$

${ }_{95} \mathrm{~Hz}, 7.0181 \times 10^{14} \mathrm{~Hz}$, and $7.3074 \times 10^{14} \mathrm{~Hz}$, respectively. We can see that the SRRs produce strong electric resonance and highly localized electric field energy at the gap is coupled to the hole except the cut of SRRs. Besides, there is relatively rather strong resonance in $\mathrm{SRR}_{A}$ (the split rings of $\mathrm{A}$ ), which contributes to the interaction of multiple electric and magnetic dipoles enhancement. The other enhancement is mainly due to the resonance of $\mathrm{SRR}_{B}$ (the split rings of B), as show in Fig. 2(b). 
The electric field distribution of hollowed-out SRRs in this paper is much different from the traditional SRRs. Ref. [15, 24, 25] The traditional SRRs, which the electric field distribution focused on the splits, and most of the other place the electric field is almost zero. Inversely, the electric field of the hollowedout SRRs is distributed on the gap of the SRRs. The intensity of electric field is stronger than the traditional structure obviously

From Fig. 2(b), group $\mathrm{SRR}_{A}$ and group $\mathrm{SRR}_{B}$ have the same electric field distribution respectively, but group $\mathrm{SRR}_{A}$ is different with group $\mathrm{SRR}_{B}$. The strength and weakness of the electric field distribution alternates from group A to group B. This is due to rotation of the electric field vector $(E)$ of circular polarization wave in the plan of vertical wave vector $(k)$ direction. With the increases of frequency, the interaction of coupling from metal film before polyimide and after is increases. The directions of splits are different before polyimide and after, but they have own distribution rule. With the increases of strengthen of the electric field, the interaction of coupling from metal film before polyimide and after is obviously. The electric field of before will be affected by after, and redistributes the electric field, appears more strong and weak areas. As shown the strongest electric field distribution areas of the group B SRRs 120 from $1\left(f_{2}\right), 2\left(f_{4}\right)$ to $3\left(f_{7}\right)$ in Fig.2 (b).

\section{SIMULATION RESULTS AND DISCUSSION}

\subsection{Optimization of CMMs structure}

According to the effective media theory, the effective EM parameters could be retrieved from the complex scattering parameters (S-parameters, include transmission $T$ and refraction coefficients $R$ ). Ref. [18] The simulation has been performed based on the standard finite-difference time domain (FDTD) method. The periodic boundary conditions are applied to the $\mathrm{x}$ and $\mathrm{y}$ directions and the absorbing boundary conditions to the $\mathrm{z}$ direction as well. Based on above numerical simulation method, the final calculated S-parameters of 130 multiple samples as shown by Fig. 3(a)-(c). 

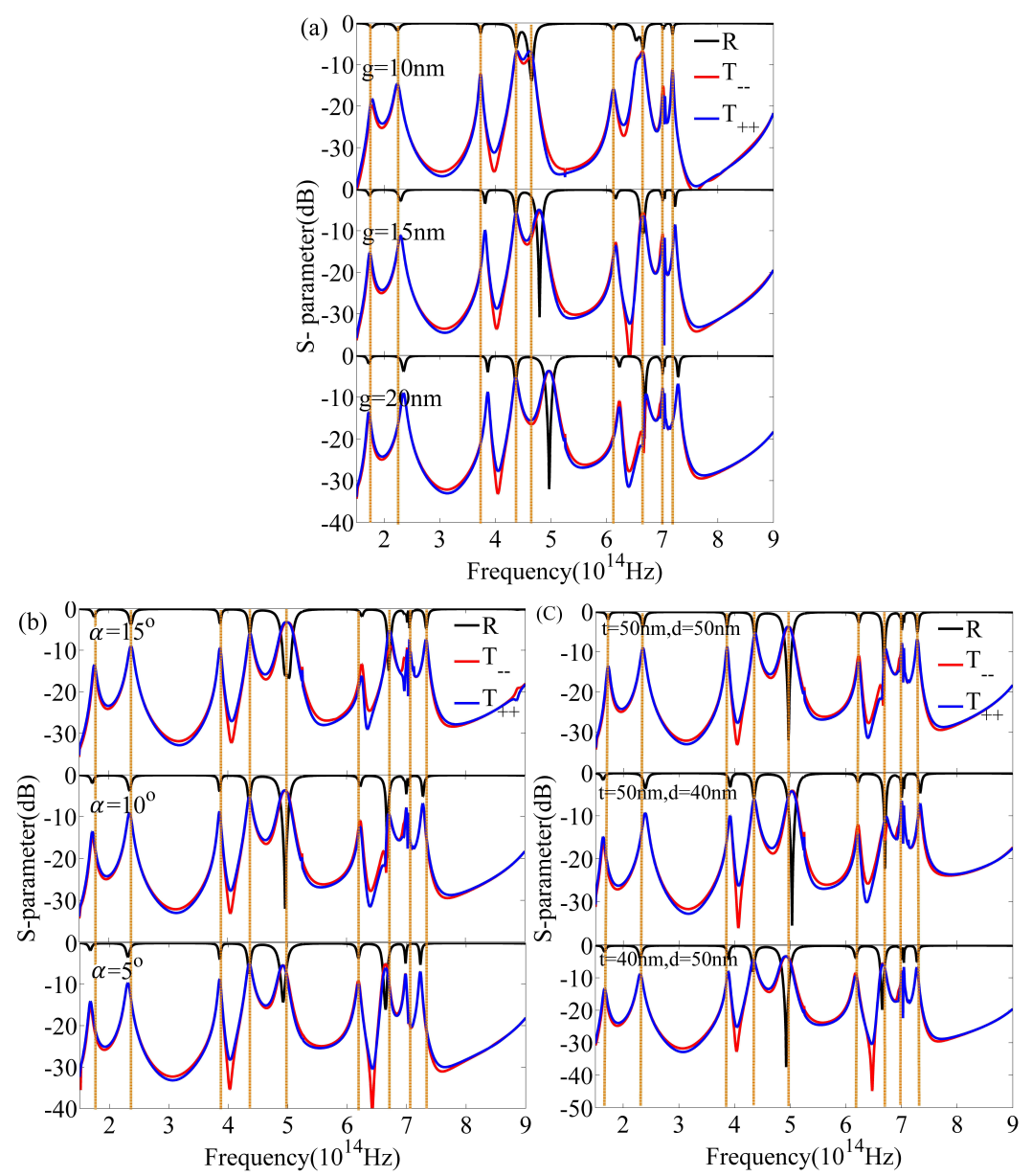

Fig. 3. S-parameters of multiple samples.

For the purpose of comparison, as shown in Fig.3 $\alpha, t$ and $d$ remain while $g$ is adjusted. The CMM has nine resonance frequencies (transmission peaks shown by yellow lines). This indicates that the resonance frequency regions 135 will blue-shift with the increase in $g$, and the reflection coefficient $R$ decreases significantly with the increase of $g$ at about $5 \times 10^{14} \mathrm{~Hz}$ and $6.5 \times 10^{14} \mathrm{~Hz}$. The reflection coefficient $R$ and transmission coefficients $T_{++} / T_{--}$show that when the $R$ is at its minimum, $T_{++} / T_{--}$reach their maxima. Furthermore, the bands around resonance frequencies become wider with the increasing of $g$, 
which results in superior transmission for the visible light region. Under the impact of chirality, a difference exists between $T_{++}$and $T_{--}$, which indicates that the chiral structure causes different transmissions in the RCP and LCP waves. In particular, at about $4 \times 10^{14} \mathrm{~Hz}, T_{++}$is nearly $10 \mathrm{~dB}$ higher than $T_{--}$. Furthermore, $T_{++}$reversal smaller than $T_{--}$at about $6.3 \times 10^{14} \mathrm{~Hz}$ with 145 the increase in $g=20 \mathrm{~nm}$. This sufficiently reflects the optical spatial dispersion of the CMMs.

As shown in Fig.3(b), $g, t$ and $\mathrm{d}$ remains constant while $\alpha$ is adjusted. Continue to the reduction of $\alpha$, there is no generate movement is found in the resonance frequencies (unlike the case of the increase in $g$ ), though the $R$ curve was found to increase. The structure is designed as a hollow structure, if the hollow area is large, EMW transmittance should be maximal and reflection should be minimal. For example, if $g$ is increased, then EMW transmittance is improved. However, when we reduce the cut angle $\alpha$ in order to further increase the size of the hollow area, we find that EMW transmittance is not improved. ${ }_{155}$ As shown in Fig. 3(b), the $R$ curve is at a minimum and $T_{++} / T_{--}$are at their maxima at $4.9638 \times 10^{14} \mathrm{~Hz}$ and $6.6854 \times 10^{14} \mathrm{~Hz}$, respectively, when $g=20$ $\mathrm{nm}$ and $\alpha=10^{\circ}$. This is because each ring can be regarded as an LC oscillatory circuit, thereby forming an electric dipole for the transmission of electric energy, the coupling of adjacent rings forms a magnetic dipole for the transmission of magnetic energy. Under these conditions, the magnetic field energy is $L Q^{\prime 2} / 2$ and the electric field energy is $Q^{2} / 2 C[5]$. When $\alpha$ and $g$ are increased, this makes the inductance $L$, capacitance $C$, and the electrical charge $Q$ all increase, which means that the magnetic field energy and electric field energy increase as well. The transmission energy is equal to the sum of the electric and magnetic field energies; therefore, $T$ increases as $R$ decreases. This result is consistent with the results of the previously performed qualitative analysis. When $g$ and $\alpha$ are all decreased, it makes $L$ decrease, $C$ and $Q$ increase. Accordingly, the electric field energy increases but the magnetic field energy decreases, and the transmission potential increases non-monotonically. Therefore, when the ring cut $\alpha$ decreases, the transmission coefficient will increase initially and then later 
decrease.

By adjusting $g$ and $\alpha$, we found that $g$ determines the resonance frequencies and that the EMW transmittance increases with $g$. However, $\alpha$ does not affect the resonance frequencies, but affects the EMW transmittance only to a certain extent. In summary, the optimal parameters of this structure should be $g=20$ $\mathrm{nm}$ and $\alpha=10^{\circ}$.

While maintaining the parameters at $g=20 \mathrm{~nm} \alpha=10^{\circ}$ (along with the size of the unit cell), the parameters $t$ and $d$ were then adjusted. We obtained Sparameters of multiple samples through the simulation for $t=50 \mathrm{~nm}$ and $d=50$ $\mathrm{nm}, t=50 \mathrm{~nm}$ and $d=40 \mathrm{~nm}$, as well as $t=40 \mathrm{~nm}$ and $d=50 \mathrm{~nm}$, as shown in Fig. $3(\mathrm{c})$. According to this figure, the dielectric medium thickness d has no effect on the S-parameter, whereas the metal thickness $t$ has a significant effect on $T_{--}$ in the high-frequency region. At $t=40 \mathrm{~nm}$, we can also see the decrease of $T_{--}$ occur only in the high-frequency region $\left(6.465 \times 10^{14} \mathrm{~Hz}\right), T_{--}$decreases nearly $20 \mathrm{~dB}$, but $T_{++}$remain unchanged, which illustrates an increase in circular dichroism. And in lower frequency $\left(4.035 \times 10^{14} \mathrm{~Hz}\right) T_{--}$remain the value with the other samples. That is because of the influence of the metal skin depth in high-frequency regions. Furthermore, in high-frequency regions, the metals absorption of EMWs is influenced by the metal size: smaller values of $t$ lead to a higher absorption of EMWs.

\subsection{Results and discussion}

On the basis of Eqs. (1)-(3), $\Delta, \theta$, and $\eta$ are shown in Fig. 4(a)and(b).

As shown in Fig. 4(a), $\alpha$ remains unchanged at $10^{\circ}$, while $g$ is adjusted. In Fig. 4(b), $g$ remains unchanged at $20 \mathrm{~nm}$ while $\alpha$ is adjusted. On the basis of the increase $g$ and $\alpha$, the resonance regions around $5 \times 10^{14} \mathrm{~Hz}$ move to blue-shifts obviously. A significant increase in $\mathrm{CD}(\Delta)$ indicates a big difference between transmission spectra, and two significant peaks. This result indicates that the chiral structure has good CD in the visible light region, and that the increase in ring width $g$ and ring cut $\alpha$ can improve the CD of the CMM.

$\theta$ refers to the polarized angle of rotation of the exiting wave polarization 
plane against the incident wave per unit length. When $g=10 \mathrm{~nm}, \alpha=10^{\circ}$ and $g=20 \mathrm{~nm}, \alpha=15^{\circ}$ (decrease hollow area), $\theta$ is up to maximal value of $180^{\circ}$, higher than that of other chiral negative refraction metamaterials in infrared and visible light regions. Ref. 15] Therefore, the proposed structure has improved optical activity. refers to the change in the angle of the exiting wave against the polarization of the incident wave, as shown in Fig. 4(a) and(b).

These results indicate that the polarization of the incident wave of the CMM has changed at the points of resonance frequency. The regulation of the chiral structure parameters on $\eta$ is similar to the irregulation on $\Delta$. $\eta$ is significantly small throughout the whole frequency range (in most of the frequency regions, $\eta$ is zero). This illustrates the extremely small ellipticity of the transmitted wave, which can be used to minimize the polarization distortion and achieve high transmission. In this way, one can utilize the off-resonance frequency regions of the bilayer CMM response.

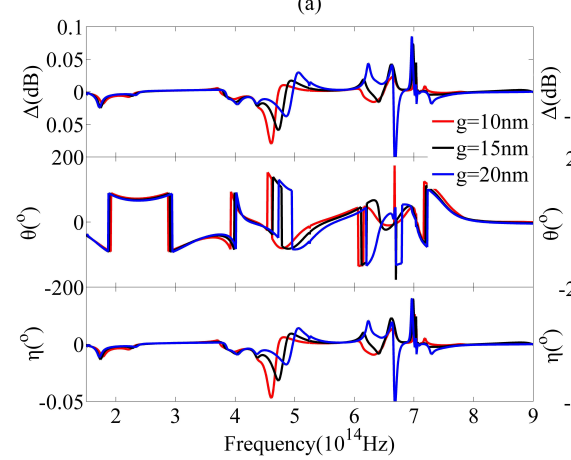

Fig. 4. Optical properties of multiple samples. (b)

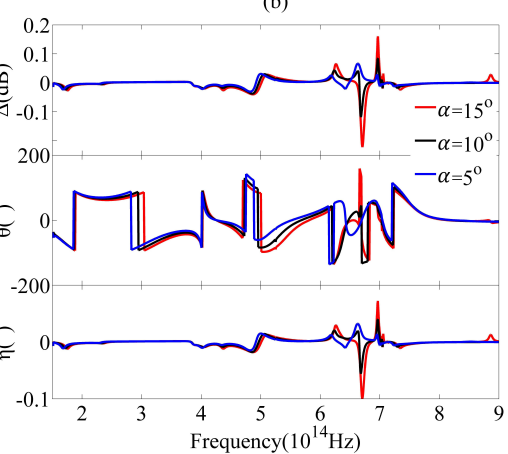

On the basis of Eqs. (5)-(7), $\kappa_{e f f}, n_{-}, n_{+}$, and $n_{e f f}$ of the chiral structure are shown in Fig. 5. In Fig. 5(a), $\alpha$ remains unchanged at $10^{\circ}$ while $g$ is adjusted. In Fig. 5(b), $g$ remains constant at $20 \mathrm{~nm}$ while $\alpha$ is adjusted. 
As shown in Figs. 5(a) and (b), the extreme value of the equivalent parameter $\kappa_{\text {eff }}$ occurs at the resonance frequencies, and a stable value is maintained in certain frequency regions. The regulation of ring width $g$ and ring cut $\alpha$ change only the resonance frequency instead of significantly regulating the value of $\kappa_{\text {eff }}$. This is mainly because the size change we selected is small and the chiral structure basically does not change.

As shown by the $n_{-}$in Figs. 5(a) and (b), the chiral structure has five negative refraction bands in the simulation frequency range of $1.5 \times 10^{14} \mathrm{~Hz}$ to $9 \times 10^{14} \mathrm{~Hz}$. Each region of negative refractive index covers a large frequency range. For example, for $g=20 \mathrm{~nm}$ and $\alpha=10^{\circ}$, the negative refractive index frequency bands are as follows: $1.95 \times 10^{14} \mathrm{~Hz}$ to $2.4675 \times 10^{14} \mathrm{~Hz} ; 4.035 \times 10^{14}$ $\mathrm{Hz}$ to $4.1775 \times 10^{14} \mathrm{~Hz} ; 4.755 \times 10^{14} \mathrm{~Hz}$ to $4.965 \times 10^{14} \mathrm{~Hz} ; 6.975 \times 10^{14} \mathrm{~Hz}$ to $7.035 \times 10^{14} \mathrm{~Hz}$; and $7.425 \times 10^{14} \mathrm{~Hz}$ to $7.785 \times 10^{14} \mathrm{~Hz}$, accounting for $18 \%$ of the total stimulation frequency range and for $22 \%$ of the visible light region. The regulation of ring width $g$ and ring cut $\alpha$ has a certain regulating effect on the negative refraction. Increasing the ring width $g$ blue-shifts the whole negative index regions. The index of refraction is -2.5 . The regulation of ring cut $\alpha$ has a minimal impact on negative refraction frequency range and value.

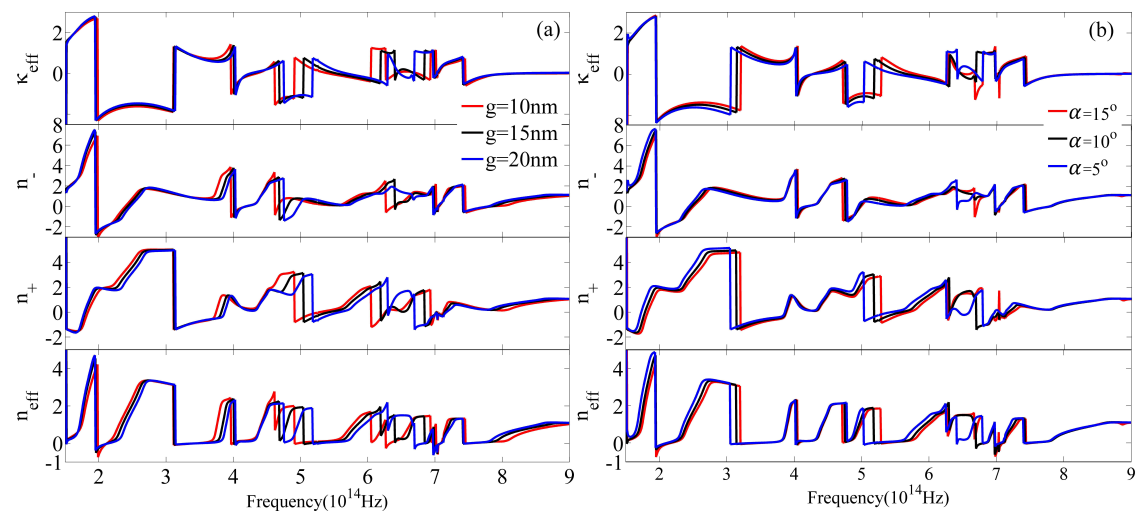

Fig. 5. Chiral parameter and equivalent refractive index of multiple samples. 
As shown by the $n_{+}$in Figs. 5(a) and (b), the chiral structure has five negative refraction bands in the simulation frequency range of $1.5 \times 10^{14} \mathrm{~Hz}$ to $9 \times 10^{14} \mathrm{~Hz}$. Each region of negative refractive index covers a large frequency range. Similarly, for $g=20 \mathrm{~nm}$ and $\alpha=10^{\circ}$, the negative refractive index frequencies are as follows: $1.515 \times 10^{14} \mathrm{~Hz}$ to $1.785 \times 10^{14} \mathrm{~Hz}, 3.1425 \times 10^{14} \mathrm{~Hz}$ to $3.87 \times 10^{14} \mathrm{~Hz}, 5.1825 \times 10^{14} \mathrm{~Hz}$ to $5.58 \times 10^{14} \mathrm{~Hz}, 6.30 \times 10^{14} \mathrm{~Hz}$ to $6.315 \times 10^{14}$ $\mathrm{Hz}$, and $6.6975 \times 10^{14} \mathrm{~Hz}$ to $7.17 \times 10^{14} \mathrm{~Hz}$, accounting for $26 \%$ of the total stimulation frequency range and for $25 \%$ of the visible light frequency region. The extreme value of $n_{+}$is -1.9 .

As shown in Figs.5(a) and (b), $\kappa_{e f f}$ has nine equivalent negative refractive index bands, among which there are eight broad negative refractive index bands, as follows: $1.95 \times 10^{14} \mathrm{~Hz}$ to $2.1375 \times 10^{14} \mathrm{~Hz}, 3.1425 \times 10^{14} \mathrm{~Hz}$ to $3.4575 \times 10^{14} \mathrm{~Hz}$, $4.03 .5 \times 10^{14} \mathrm{~Hz}$ to $4.05 \times 10^{14} \mathrm{~Hz}, 5.1825 \times 10^{14} \mathrm{~Hz}$ to $5.235 \times 10^{14} \mathrm{~Hz}, 5.25 \times 10^{14}$ Hz to $5.28 \times 10^{14} \mathrm{~Hz}, 6.69 \times 10^{14} \mathrm{~Hz}$ to $6.8325 \times 10^{14} \mathrm{~Hz}, 6.975 \times 10^{14} \mathrm{~Hz}$ to $7.08 \times 10^{14}$ $\mathrm{Hz}$, and $7.425 \times 10^{14} 4 \mathrm{~Hz}$ to $7.4925 \times 10^{14} \mathrm{~Hz}$, accounting for $14 \%$ of the total stimulation frequency range and for $12 \%$ of the visible light frequency range. The number and width of frequency bands of this negative refraction CMM in the visible light region are far better than those of the fishnet structure negativeindex material discussed in the literature. Ref. [26]

As shown in Fig.5, the change in structural parameters has a regulating effect on the negative refraction, wherein the resonance frequencies blue-shift with increases in the ring width $g$ and ring cut $\alpha$.

A longitudinal comparison of $\kappa_{e f f}, n_{-}, n_{+}$, and $n_{e f f}$ shows that $n_{-}$appears negative for the negative minimum value of the chiral parameter, and continues negative in a certain range that the chiral parameter maintains lower value of the chiral parameter. $n_{+}$appears in the negative maximum value of the chiral parameter, and continues negative in a certain range that the chiral parameter maintains higher value of the chiral parameter. These results indicate that the chiral structure has a promoting effect and an inhibiting effect on the LCP and RCP waves, respectively. $\kappa_{\text {eff }}$ is represented in a certain extreme value that the chiral parameter maintains; whether it is negative or not further depends 
270 on the values of $n_{-}$and $n_{+}$.

\section{Conclusion}

In summary, we have designed and optimized a conjugated bilayer metal four-cut split-ring resonator in the infrared and visible light regions. The S-parameter obtained in the frequencies of $1.5-9 \times 10^{14} \mathrm{~Hz}$ has nine resonance regions. The retrieval calculation shows that the LCP and RCP waves in this frequency range have five negative refractive index frequency bands and eight broad equivalent negative refraction frequency bands, respectively, with a higher and wider frequency range than the CMMs in the existing literature. Ref. 25] With regard to the visible light range, the negative refraction bands of the ${ }_{280} \mathrm{LCP}$ wave, the RCP wave, and the equivalent account for $22 \%, 25 \%$, and $12 \%$ of total visible light region, respectively. Meanwhile, the number of negative refraction bands is more than that reported in the literature. Ref. [15, 24] The increase in ring width $g$ and ring cut $\alpha$ can regulate the resonance frequencies by blue-shifting them, thereby optimizing the negative refraction and optical

285 activity. We believe that $g=20 \mathrm{~nm}$ and $\alpha=10^{\circ}$ represent the best parameters for this CMM. This CMM has a large optical activity, and a high angle of rotation (i.e., up to $180^{\circ}$ ). We also analyzed the resonance mechanism of this chiral structure for achieving eight bands of negative refraction. We believe that the joint action of so many electric and magnetic dipoles is an important factor for 290 achieving multi-band negative refraction. We believe that this CMM will be significantly applied in functional materials, optical devices, and other fields.

\section{ACKNOWLEDGMENTS}

This work was supported by the National Natural Science Foundation of China (No 61205011)and the National Science Foundation of China (NSFC) (No. 61205093). 


\section{References}

[1] D. C. V. S. C. N.-N. D. R. Smith, W. J. Padilla, S. Schultz, Composite medium with simultaneously negative permeability and permittivity, Appl. Phys. Lett 84 (2000) 4184.

[2] A. S. S. M. S. Tretyakov, I. Nefedov, C. Simovski, Waves and energy in chiral nihility, J. Electromagn. Waves Appl. 17 (2003) 695.

[3] J. B. Pendry, A chiral route to negative refraction,, Science 306. (2004) 1353.

[4] A. S. S. A. V. Rogacheva, V. A. Fedotov, N. I. Zheludev, Giant gyrotropy due to electromagnetic-field coupling in a bilayered chiral structure, Phys. Rev. Lett. 97 (2006) 177401.

[5] L. G. Fang Fang, H. Liao, Pseudo-planar conjugated swastikas metamaterial with giant circular dichroism and negative refraction at near-infrared region, Journal of Modern Optics 63 (2016) 190-194.

[6] T. L. S. M. W. F. M. W. R. X. W. P. C. S. N. Z. T. Q. Li, H. Liu, X. Zhang, Magnetic resonance hybridization and optical activity of microwaves in a chiral metamaterial, Appl. Phys. Lett. 92 (2008) 131111.

[7] S. L. M. Decker, M. Wegener, Coupling effects in low-symmetry planar split-ring resonator arrays, Opt. Lett. 34 (2009) 1579.

[8] T. K. B. Wang, C. Soukoulis, Wide-angle and polarizationindependent chiral metamaterial absorber, Phys. Rev. B 033108 (2009) 80.

[9] W. L. Z. R. Singh, E. Plum, N. I. Zheludev, Highly tunable optical activity in planar achiral terahertz metamaterials, Opt. Express 18 (2010) 13425.

[10] M. K. B. Wang, T. Koschny, C. M. Soukoulis, Chiral metamaterials: Simulations and experiments 11 (2009) 114003. 
[11] A. S. M. Mutlu, A.E. Akosman, E. Ozbay, Asymmetric transmission of linearly polarized waves and polarization angle dependent wave rotation using a chiral metamaterial, Opt. Express 19 (2011) 14290.

[12] B. W. T. K. M. K. J. Zhou, J. Dong, C. M. Soukoulis, Negative refractive index due to chirality, Phys. Rev. B 79 (2009) 121104.

[13] T. K. J. Dong, J. Zhou, C. Soukoulis, Bi-layer crosschiralstructure with strong optical activity and negative refractive index, Opt.Express 17 (2009) 14172 .

[14] Q. H. F. Y. H. L. K. Song, X. P. Zhao, W. R. Zhu, Wide-angle $90^{\circ}-$ polarization rotator using chiral metamaterial with negative refractive index, J. Electromagnet. Wave 26 (2012) 1967.

[15] C. S. W. Panpradit, A. Sonsilphong, N. Wongkasem, High negative refractive index in chiral metamaterials, J. Opt. 14 (2012) 075101.

[16] A. S. S. Tretyakov, L. Jylhä, Backward-wave regime and negative refraction in chiral composites, Photon. Nanostruct. Fundam. Appl. 3 (2005) 107.

[17] J. D. V. A. F. T. K. C. M. S. E. Plum, J. Zhou, N. I. Zheludev, Metamaterial with negative index due to chirality, Phys. Rev. B 79 (2009) 035407.

[18] B. W. J. P. J. X. Chen, M. Tomasz. Grzegorczyk, J. Kong, Robust method to retrieve the constitutive effective parameters of metamaterials, Phys. Rev. B 70 (2004) 016608.

[19] E. C. Z. Li, K. B. Alici, E. Ozbay, Complementary chiral metamaterials with giant optical activity and negative refractive index, Appl. Phys. Lett. 98 (2011) 161907.

[20] M. A. G. L. J. D. B. J. B. M. B. R. R. M. F. F. M. F. Falcone, T. L. Opetegi, M. Sorolla, Babinet principle applied to the design of metasurfaces and metamaterials, Phys. Rev. Lett. 93 (2004) 19740. 
[21] A. S. S. K. T. Zentgraf, T. P. Meyrath, H. Giessen, Babinet/s principle for optical frequency metamaterials and nanoantennas, Phys. Rev. B 76 (2007) 033407.

[22] S. K. N. Liu, H. Giessen, Magnetoinductive and electroinductive coupling in plasmonic metamaterial molecules, Adv. Mater. 20 (2008) 4521.

[23] J. L. X. L. W. Z. S. Zhang, Y. S. Park, X. Zhang, Negative refractive index in chiral metamaterials, Phys. Rev. Lett. 102 (2009) 023901.

[24] V. V. V. A. Lakhtakia, V. K. Varadan, Eigenmodes of a chiral sphere with a perfectly conducting coating, J. Opt. Soc. Am. A 5 (1988) 175.

[25] W. Q. Menga, B. Zenga, B. Zhang, Enhancement effect of terahertz wave in split ring resonators, Proc. of SPIE. 8841 (2013) 884112.

[26] B. K. M. Choi, J. Choe, C. Choi, A flexible metamaterial with negative refractive index at visible wavelength, Curr. Appl. Phys. 13 (2013) 1723. 